\title{
Schistosoma mansoni Infection and Associated Determinant Factors among School Children in Sanja Town, Northwest Ethiopia
}

\author{
Ligabaw Worku, ${ }^{1}$ Demekech Damte, ${ }^{1}$ Mengistu Endris, \\ ${ }^{1}$ Habtie Tesfa, ${ }^{1}$ and Mulugeta Aemero ${ }^{2}$ \\ ${ }^{1}$ School of Biomedical and Laboratory Sciences, University of Gondar, 196 Gondar, Ethiopia
}

${ }^{2}$ Department of Biology, University of Gondar, 196 Gondar, Ethiopia

Correspondence should be addressed to Ligabaw Worku; ligabaw@gmail.com

Received 29 October 2013; Revised 18 January 2014; Accepted 27 February 2014; Published 1 April 2014

Academic Editor: Maria V. Johansen

Copyright (c) 2014 Ligabaw Worku et al. This is an open access article distributed under the Creative Commons Attribution License, which permits unrestricted use, distribution, and reproduction in any medium, provided the original work is properly cited.

Background. Intestinal schistosomiasis is one of the most widespread parasitic infections in tropical and subtropical countries. Objective. To determine the prevalence of $S$. mansoni infection and associated determinant factors among school children in Sanja Town, northwest Ethiopia. Methods. A cross-sectional study was conducted from February to March, 2013.385 school children were selected using stratified proportionate systematic sampling technique. Pretested questionnaire was used to collect sociodemographic data and associated determinant factors. Stool samples were examinedusing formol-ether concentration and Kato-Katz technique. Data were entered and analyzed using SPSS 20.0 statistical software. Multivariate logistic regression was done for assessing associated risk factors and proportions for categorical variables were compared using chi-square test. $P$ values less than 0.05 were taken as statistically significant. Results. The prevalence of $S$. mansoni infection was $89.9 \%(n=346)$. The overall helminthic infection in this study was $96.6 \%(n=372)$. Swimming in the river, washing clothes and utensil using river water, crossing the river with bare foot, and fishing activities showed significant association with the occurrence of $S$. mansoni infection. Conclusion. Schistosoma mansoni infection was high in the study area. Therefore, mass deworming at least twice a year and health education for community are needed.

\section{Background}

Schistosomiasis is one of the most widespread parasitic infections in developing countries [1]. An estimated 779 million people are at risk, with 240 million infected cases and more than 200,000 deaths occurring each year due to schistosomiasis worldwide [2,3]. In Africa, over $90 \%$ of disease burden is found in sub-Saharan Africa [4], where S. mansoni and $S$. haematobium are the main causative species of schistosomiasis in the continent with an estimation of 54 and 112 million individuals infected, respectively, and the risk of infection for S. mansoni and S. haematobium was 393 and 436 million, respectively [5].

Schistosomiasis is also one of the most common parasitic diseases and is widespread in many parts of Ethiopia, usually at an altitude between 1,200 and 2,000 meters above sea level [6]. Many reports show that intestinal schistosomiasis caused by $S$. mansoni is widely distributed in the country than urinary schistosomiasis caused by $S$. haematobium [7-15]. The main determinants for the distribution, transmission, and spreading of both Schistosoma species (S. mansoni and $S$. haematobium) in Ethiopia include water temperature, absence or presence of snail intermediate host, population movement, and water impoundment for irrigation and power [9].

Schistosomiasis affects human host by slow damage of the host organs due to granuloma formation around the eggs in the tissues. This leads to the development of fibrosis and chronic inflammation in the liver and causes severe damage including bleeding, renal failures, and cancer [16].

The high prevalence of this infection is closely correlated to infested water bodies (pond, stream, river, and dam) 
contact during crossing with bare foot, swimming, washing of clothes and utensil, playing, fishing, and irrigation activity [17]. School-aged children are the most affected group due to high exposure to infested water bodies. Growth retardation and poor school performance are adverse effects of the disease besides clinical manifestation and its complication [18]. Although several studies have been conducted on the prevalence of $S$. mansoni and their risk factors in Ethiopia, there is still a lack of epidemiological information in some localities of northwest Ethiopia. Therefore, the aim of this study was to determine the prevalence of $S$. mansoni infection and associated determinant factors among school children of Sanja General Elementary School in Sanja Town, northwest Ethiopia.

\section{Methods}

2.1. Study Design and Period. A cross-sectional study was conducted from February 1 to March 30, 2013, at Sanja Town, northwest Ethiopia.

2.2. Study Area. Sanja Town is located $792 \mathrm{~km}$ far from the capital city Addis Ababa on the roadside to GondarHumera. Sanja has an altitude of $1800 \mathrm{~m}$ above sea level with annual rainfall ranging from 800 to $1800 \mathrm{~mm}$ and annual temperature ranging from $25^{\circ} \mathrm{C}$ to $42^{\circ} \mathrm{C}$. There are two elementary schools, one junior and one high school. One health center gives service for the dwellers of the town and the surrounding areas. There is a river known as Sanja that flows throughout the year. Sanja General Elementary School is located on the west of the main road. A total of 2079 (872 male, 1207 female) students were enrolled in the school for 2012/13 academic year.

2.3. Study Population. The study populations were Sanja General Elementary School children who were enrolled in the school during the study period.

2.4. Sampling and Sample Size Determination. The sample size was determined using statistical formula $\left(n=\left(z^{2} p(1-\right.\right.$ $p) d^{2}$ ) considering 95\% confidence interval and 50\% prevalence. Based on this assumption, 385 study participants were selected from 2079 students. To select the study subjects, the students were first stratified according to their educational level (Grade 1 to Grade 8). Allocation of student was proportional to the number of students in each grade. Finally, the school children were selected using systematic sampling using class roster as the sampling frame. Every fifth pupil was selected for the study.

\subsection{Data Collection}

2.5.1. Sociodemography and Determinant Factors Assessments. Sociodemography and possible determinant factors were assessed with pretested and standardized questionnaire, which was translated into the local language, Amharic.
2.5.2. Detection and Quantification of Intestinal Helminths. The school children were provided with small, clean, dried, and leak proof container and clean wooden applicator stick. Then, they were informed to bring sizable stool sample of their own. Then, the stool sample was processed and diagnosed microscopically using formol-ether concentration and Kato-Katz techniques. Double Kato-Katz was employed to a template delivering $41.7 \mathrm{mg}$ of stool [19]. Eggs counted for $S$. mansoni and other common intestinal helminths were recorded and later converted into eggs per gram of stool (EPG), multiplying by a factor of 24 . Infection intensity (light, moderate, and high) was classified according to the World Health Organization (WHO) criteria [20].

2.5.3. Quality Control. All the necessary reagents, chemicals, and the performance of kits were checked by known positive and negative samples before processing and examination of samples of the study subjects. The data was checked for completeness and any incomplete or misfiled questionnaires were recorrected under supervision. The slides were examined by two microscopists independently under the middle (40x) objective of the microscope. Negative samples were reexamined on the same day at the same time by another laboratory technologist. The result of laboratory examination was recorded on well-prepared format carefully. Ten percent of the total slide was randomly selected and reexamined at the end by experienced laboratory technologist at the University of Gondar, who was blinded for the first examination results.

2.5.4. Data Analysis and Interpretation. Data were entered and analyzed using SPSS 20.0 (SPSS Inc., Chicago, 2011) software. Descriptive statistics was used to give a clear picture of background variables. The frequency distribution of both dependent and independent variables was worked out. Multivariate logistic regression was done for assessing associated risk factors and proportions for categorical variables were compared using chi-square test. $P$ values less than 0.05 were taken as statistically significant.

2.5.5. Ethical Considerations. Ethical clearance was obtained from research and ethics review committee of School of Biomedical and Laboratory Sciences, University of Gondar. Before starting of the actual data collection, permission was obtained from school director. Additionally, after explaining the importance of the study, an informed written consent was obtained from study participant's parent/guardian. An assent was also taken from the school children. Those children who were positive for $S$. mansoni and other intestinal parasites were treated according to the national protocol. 
TABLE 1: Sociodemographic characteristics of school children in Sanja General Elementary School, Sanja, northwest Ethiopia, 2013.

\begin{tabular}{lcc}
\hline Sociodemographic characteristics & Number & Percent \\
\hline Sex & & \\
Male & 132 & 34.3 \\
Female & 253 & 65.7 \\
Age (years) & & \\
6-10 & 73 & 19 \\
11-15 & 312 & 81 \\
Residence & & \\
Rural & 97 & 25.2 \\
Urban & 288 & 74.8 \\
Mothers/guardian educational status & & \\
Illiterate & 144 & 37.4 \\
Read and write & 153 & 39.7 \\
Primary school & 62 & 16.1 \\
Secondary school and above & 26 & 6.8 \\
Respondent grade & & \\
One & 46 & 11.9 \\
Two & 30 & 7.8 \\
Three & 27 & 7.0 \\
Four & 25 & 6.5 \\
Five & 47 & 12.2 \\
Six & 69 & 17.9 \\
Seven & 64 & 16.6 \\
Eight & 77 & 20.0 \\
\hline Total & & 100 \\
\hline
\end{tabular}

TABle 2: Prevalence of $S$. mansoni and common intestinal helminths by sex among school children in Sanja General Elementary School, Sanja, northwest Ethiopia, 2013.

\begin{tabular}{lccccc}
\hline Parasite & Male & Female & Total & P value & $\chi^{2}$ \\
& $N=132$ & $N=253$ & $N=385$ & & \\
\hline S. mansoni & $119(34.4)^{* *}$ & $227(65.6)$ & $346(89.9)$ & 0.895 & 0.02 \\
Hookworm & $49(34.3)$ & $94(65.7)$ & $143(37.1)$ & 0.995 & 0.00 \\
A. lumbricoides & $24(38.7)$ & $38(61.3)$ & $62(16.1)$ & 0.423 & 0.46 \\
T. trichiura & $3(100)$ & $0(0)$ & $3(0.8)$ & $0.016^{*}$ & 5.80 \\
Taenia species & $0(0)$ & $6(100)$ & $6(1.6)$ & 0.075 & 3.18 \\
H. nana & $3(41.9)$ & $18(58.1)$ & $31(8.1)$ & 0.47 & 3.94 \\
\hline Total & $124(33.3)$ & $248(66.7)$ & $372(96.6)$ & $0.035^{*}$ & 4.44 \\
\hline
\end{tabular}

${ }^{*}$ The difference was statistically significant $(P<0.05)$.

${ }^{* *}$ Figures in parenthesis indicate percentages.

\section{Results}

3.1. Sociodemographic Characteristics. A total of 385 school children were included in the study. Their mean age was 12.7 years (range: 6 to 15 years) with standard deviation of 2.3. Among these, 132 (34.3\%) were males and $253(65.7 \%)$ were females. Most of the school children 312/385 (81\%) were between 11 and 15 years. Of the 385 school children, 288 (74.8\%) were from Sanja Town while 97 (25.2\%) were from the surrounding rural areas (Table 1).
TABLE 3: The intensity of Schistosoma mansoni and common intestinal helminths using Kato-thick smear technique among Sanja General Elementary School children, northwest Ethiopia, 2013.

\begin{tabular}{lccc}
\hline Parasite & Light & $\begin{array}{l}\text { Intensity } \\
\text { Moderate }\end{array}$ & Heavy \\
\hline S. mansoni & $71(18.4)^{* *}$ & $181(47)$ & $72(18.7)$ \\
A. lumbricoides & $25(6.5)$ & $0(0)$ & $0(0)$ \\
Hookworm & $29(7.5)$ & $0(0)$ & $0(0)$ \\
T. trichiura & $1(0.3)$ & $0(0)$ & $0(0)$ \\
\hline Total & $126(32.7)$ & $181(47)$ & $72(18.7)$ \\
\hline ** $^{*}$ Tigures in parenthesin
\end{tabular}

${ }^{* *}$ Figures in parenthesis indicate percentages.

3.2. Prevalence and Egg Intensity of S. mansoni Infection. Among the total 385 school children examined, 372 (96.6\%) were positive for $S$. mansoni and/or one or more other intestinal helminths. The most prevalent parasitic infection was intestinal schistosomiasis (S. mansoni) 346 (89.9\%), followed by Hookworm 143 (37.1\%), Ascaris lumbricoides 62 (16.1\%), Hymenolepis nana 31 (8.1\%), Taenia species 6 (1.6\%), and Trichuris trichiura $3(0.8 \%)$. Of the positive cases, $66.7 \%$ were females and $33.3 \%$ were males (Table 2).

Regarding parasitic load, the highest number of egg count per gram of faeces (EPG) for S. mansoni was 1776, which was observed only in one student. The intensity of infection using the Kato-Katz method among the total parasite infected school children 126 (32.7), 181 (47), and 72 (18.7\%) was heavy, moderate, and heavy, respectively, whereas the intensity of S. mansoni infection 71 (18.4), 181 (47), and 72 (18.7\%) was heavy, moderate, and heavy, respectively (Table 3 ).

3.3. Determinants of S. mansoni Infection. Of the total 385 school children examined, 119/132 (90.2\%) male and 227/253 (89.7\%) female school children were positive for schistosomiasis. The distribution of S. mansoni infection among each age group showed that $90.4 \%$ of $6-10$ years and $89.7 \%$ of the 11-15 years were infected. Prevalence of S. mansoni infection among school children who lived in rural and urban areas was $88.7 \%$ and $90.3 \%$, respectively (Table 4 ).

Determinant factors assessment for S. mansoni infection in general showed that swimming in the river, frequent swimming habit, washing clothes and utensil using river water, crossing the river with bare foot, and fishing activities were associated with $S$. mansoni infection $(P<0.05)$ (Table 4).

In multivariate analysis, swimming in the river and frequent swimming habit were associated with $S$. mansoni infection $(P<0.05)$. Children who swam in the river had 5.12 times (CI: 3.47, 17.89) higher risk of being infected with S. mansoni than those who did not have a swimming habit. School children who had swimming frequency of three or more times per week were having 4.83 times (CI: 1.37, 18.81) higher odds of being infected with $S$. mansoni than those who did not (Table 4).

Schistosoma mansoni infection was also significantly associated with the habit of washing clothes and utensils. Children who wash clothes and utensils using river water 
TABLE 4: Multivariate analysis for factors potentially associated with Schistosoma mansoni infection among Sanja General Elementary School children, northwest Ethiopia, 2013.

\begin{tabular}{|c|c|c|c|c|c|c|}
\hline \multirow{2}{*}{ Risk factors } & \multicolumn{3}{|c|}{ Schistosoma mansoni number (\%) } & \multicolumn{2}{|c|}{ OR $(95 \% \mathrm{CI})$} & \multirow{2}{*}{$P$ value } \\
\hline & Positive & Negative & Total & $\mathrm{COR}$ & AOR & \\
\hline \multicolumn{7}{|l|}{ Sex } \\
\hline Male & $119(90.2)$ & $13(9.8)$ & $132(34.3)$ & $0.95(0.45,2.02)$ & $0.86(0.41,1.80)$ & \multirow{2}{*}{0.69} \\
\hline Female & $227(89.7)$ & $26(10.3)$ & $253(65.7)$ & 100 & 100 & \\
\hline \multicolumn{7}{|l|}{ Age (years) } \\
\hline $6-10$ & $66(90.4)$ & $7(9.6)$ & $73(19.0)$ & $0.93(0.36,2.32)$ & $0.63(0.18,2.20)$ & \multirow{2}{*}{0.47} \\
\hline $11-15$ & $280(89.7)$ & $32(10.3)$ & $312(81.0)$ & 100 & 100 & \\
\hline \multicolumn{7}{|l|}{ Resident } \\
\hline Rural & $86(88.7)$ & $11(11.3)$ & $97(25.2)$ & 100 & 100 & \multirow{2}{*}{0.53} \\
\hline Urban & $260(90.3)$ & $28(9.7)$ & $288(74.8)$ & $0.84(0.38,1.89)$ & $0.78(0.37,1.66)$ & \\
\hline \multicolumn{7}{|l|}{ Grade } \\
\hline One-four & $118(92.2)$ & $10(7.8)$ & $128(33.2)$ & $0.67(0.29,1.49)$ & $2.02(0.67,6.10)$ & \multirow{2}{*}{0.22} \\
\hline Five-eight & $228(88.7)$ & $29(11.3)$ & $257(66.8)$ & 100 & 100 & \\
\hline \multicolumn{7}{|c|}{ Mothers/guardian edu. status } \\
\hline Illiterate & $131(91.0)$ & $13(9.0)$ & $144(37.4)$ & $0.50(0.20,1.26)$ & $0.43(0.13,1.21)$ & \multirow{3}{*}{0.36} \\
\hline Read and write/1ry & $154(91.7)$ & $14(8.3)$ & $168(43.6)$ & $0.46(0.19,1.14)$ & $0.37(0.11,1.09)$ & \\
\hline 2ry school and above & $61(83.6)$ & $12(16.4)$ & $73(19.0)$ & 100 & 100 & \\
\hline \multicolumn{7}{|l|}{ Swimming in river } \\
\hline Yes & $106(79.7)$ & $27(20.3)$ & $133(34.5)$ & $5.09(2.37,11.1)$ & $5.12(3.47,17.89)^{\circ}$ & \multirow{2}{*}{0.01} \\
\hline No & $240(95.2)$ & $12(4.8)$ & $252(65.5)$ & 100 & 100 & \\
\hline \multicolumn{7}{|l|}{ Swimming frequency/wk } \\
\hline 0 & $70(79.5)$ & $18(20.5)$ & $88(22.9)$ & 1.00 & 1.00 & \multirow{3}{*}{0.00} \\
\hline $1-2$ & $170(90.9)$ & $17(9.1)$ & $187(48.6)$ & $2.57(1.18,5.60)$ & $1.82(0.79,4.23)$ & \\
\hline$\geq 3$ & $106(96.4)$ & $4(3.6)$ & $110(28.5)$ & $6.81(2.05,24.95)$ & $4.83(1.37,18.81)^{\bullet}$ & \\
\hline \multicolumn{7}{|l|}{ Washing clothes in river } \\
\hline Yes & $101(79.5)$ & $26(20.5)$ & $127(33.0)$ & $4.85(2.28,10.43)$ & $5.54(2.89,13.76)^{\circ}$ & \multirow{2}{*}{0.00} \\
\hline No & $245(95.0)$ & $13(5.0)$ & $258(67.0)$ & 100 & 100 & \\
\hline \multicolumn{7}{|l|}{ Washing utensil in river } \\
\hline Yes & $68(78.2)$ & $19(21.8)$ & $87(22.6)$ & $3.88(1.86,8.09)$ & $4.91(2.43,12.37)^{\circ}$ & \multirow{2}{*}{0.00} \\
\hline No & $278(93.3)$ & $20(6.7)$ & $298(77.4)$ & 100 & 100 & \\
\hline \multicolumn{7}{|c|}{ Crossing river with bare foot } \\
\hline Yes & $75(77.3)$ & $22(22.7)$ & $97(25.2)$ & $4.62(2.25,9.77)$ & $3.95(2.13,7.61)^{\bullet}$ & \multirow{2}{*}{0.00} \\
\hline No & $271(94.1)$ & $17(5.9)$ & $288(74.8)$ & 100 & 100 & \\
\hline \multicolumn{7}{|l|}{ Fishing in the river } \\
\hline Yes & $110(82.1)$ & $24(17.9)$ & $134(34.8)$ & $3.20(1.56,6.62)$ & $3.04(2.14,8.71)^{\bullet}$ & \multirow{2}{*}{0.00} \\
\hline No & $235(93.6)$ & $16(6.4)$ & $251(65.2)$ & 100 & 100 & \\
\hline Total & $346(89.9)$ & $39(10.1)$ & $385(100)$ & & & \\
\hline
\end{tabular}

-The difference was statistically significant $(P<0.05)$.

had 5.54 (CI: 2.89, 13.76) and $4.91(2.43,12.37)$ times higher odds of being infected with $S$. mansoni than those who did not, respectively. Children who cross the river with bare foot had 3.95 times (CI: 2.13, 7.61) higher risk of being infected with $S$. mansoni than those who did not cross the river. In addition, those students who had the habit of fishing were three times (CI: 2.14, 8.71) more likely to acquire S. mansoni infection (Table 3). Age, sex, residence, grade of student, and maternal/guardian education status had no statistically significant association with S. mansoni infection (Table 4).

\section{Discussion}

The prevalence rate of $89.9 \%$ S. mansoni infection was reported for the first time from the present study among school children in Sanja town. This study showed a higher prevalence rate of $S$. mansoni infection compared with other surveys conducted among school children from different parts of Ethiopia which reported prevalence ranging from $0.8 \%$ to $63 \%$ [21-33] and in other countries such as $16.5 \%$ Kenya [34], 27.8\% Uganda [35], 2.7\% Rwanda [36], 12.1\% 
Nigeria [37], and $14.4 \%$ Brazil [38]. The reason why the observed infection prevalence varies in this study from other findings might be due to (1) the difference in water contact behavior of the school children (frequency of contactinfested water), ecological distribution of intermediate host (snail), local endemicity of the parasite, and sample size; (2) the difference in altitude and the temperature, which is favorable for the development and survival of snail; (3) the difference of method employed for stool examination and the time of study; and (4) the variation in awareness regarding transmission and prevention of $S$. mansoni infection between the study participants in this and previous studies.

The intensity of $S$. mansoni infection in this study shows $18.4 \%$ light, $47 \%$ moderate, and $18.7 \%$ heavy among the total $S$. mansoni positive school children while no heavy or moderate 16 infection was observed for hookworm A. lumbricoides, and T. trichiura (Table 2), which is quite different from that reported from Azezo (light 67.8\%, moderate 19.8\%, and heavy 3.1\%) [32]; this might be due to the variation of infection rate.

The sex distribution of $S$. mansoni in the present study did not show a significant variation, which was similar to that reported from Rwanda and Nigeria [36, 37]. However, it was inconsistent with a research finding reported from different parts of Ethiopia such as Zarima, Tseda, and Gorgora in which the prevalence was slightly higher in females $[26,30$, 31 ], whereas the prevalence was higher in males reported from Adwa, Mekelle, Chiga, Amibera, and Tikur Wuha [21, $23,28,33]$. This difference might be due to similarity in water contact behavior of males and females such as swimming, crossing water bodies, washing clothes and/or utensils, and fishing in the present study.

The prevalence of $S$. mansoni showed equal distribution of infection in different age groups (6-10 and 11-15 years). This is inconsistent with reports of many researchers in different localities of Ethiopia [21-23, 26, 28] and Uganda [35] which was higher in the oldest group. Similarly, equal distribution of infection was observed in terms of residence. This is contrary to other findings conducted in Gorgora [26] and Tigray was higher in rural areas [38] while higher in urban area of Tikur Wuha [23]. This might be due to equal tendency of infested water exposure of both sex and proximity of Sanja River and other water bodies to the town and surrounding areas.

The habit of frequent contact with cercariae infested water such as swimming in the river, washing clothes and utensils using river water, crossing the river with bare foot, and fishing activities showed a statistically significant association with prevalence of $S$. mansoni infection. This is similar to the previous findings reported from (Adwa, Azezo, Zarima, Mekelle, Gorgora, and Amibera) Ethiopia, Kenya, and Rwanda [21, 26, $28,31-34,36]$. The prevalence of $S$. mansoni was higher in school children who had a habit of frequent swimming than who did not. This might be due to the presence of cercariae infected water body/ies in the surrounding of study areas.

\section{Conclusion}

Ninety percent of the school children were infected with S. mansoni. Swimming in the river, washing clothes and utensils using river water, crossing the river with bare foot, and fishing activities were the determinant factors identified in this study. Therefore, this calls the concerned bodies to take measures on the transmission of S. mansoni. Health education should be given to increase the awareness of school children about the risk of infested water contact. Application of mass deworming should be also considered for the students once in a year.

\section{Conflict of Interests}

The authors declare that there is no conflict of interests regarding the publication of this paper.

\section{Authors' Contribution}

Ligabaw Worku conceived the study, undertook statistical analysis, and drafted the initial and final paper. Mulugeta Aemero, Demekech Damte, and Mengistu Endris initiated the study and made major contributions to the study design, reviewed the initial and final drafts of the paper, and conducted data analysis. Habtie Tesfa participated in sample collection and performed laboratory diagnosis. All authors contributed to the writing of the paper and approved the submitted version of the paper.

\section{Acknowledgments}

The authors would like to thank the University of Gondar for the financial support and the Department of Medical Parasitology for providing excellent laboratory facilities for processing the stool samples. They are also thankful to staff members of Sanja Health Center for treating the infected school children and Sanja General Elementary School staff members for supporting field work. Lastly, their thanks go to all the parents/guardians for their consent and the children who participated in this study.

\section{References}

[1] C. H. King, "Parasites and poverty: the case of schistosomiasis," Acta Tropica, vol. 113, no. 2, pp. 95-104, 2010.

[2] Schistosomiasis: progress report 2001-2011 and strategic plan 2012-2020. Geneva, World Health Organization, 2012.

[3] WHO, Schistosomiasis Fact Sheet, http://www.who.int/mediacentre/factsheets/fs115/en/index.html.

[4] Working to overcome the global impact of neglected tropical diseases, World Health Organization, WHO, Geneba, Switzerland, 2010.

[5] M. J. Van Der Werf, S. J. De Vlas, S. Brooker et al., "Quantification of clinical morbidity associated with schistosome infection in sub-Saharan Africa," Acta Tropica, vol. 86, no. 2-3, pp. 125$139,2003$.

[6] C. Lo, H. Kloos, and B. Hailu, "Schistosomiasis," in The Ecology of Health and Disease in Ethiopia, Z. Ahmed and H. Kloos, Eds., Addis Ababa: EMPDA Press, 1988.

[7] H. Birrie, S. Tedla, B. Erko, N. Berhe, and F. Abebe, "Schistosomiasis in Finchaa river valley, Wellega region, West Ethiopia," 
Ethiopian Journal of Health Development, vol. 7, no. 1, pp. 9-15, 1993.

[8] H. Kloos, C. T. Lo, H. Birrie, T. Ayele, S. Tedla, and F. Tsegay, "Schistosomiasis in Ethiopia," Social Science and Medicine, vol. 26, no. 8, pp. 803-827, 1988.

[9] A. Ali, B. Erko, W. T. Micheal, and H. Kloos, "Schistosomiasis," in Epidemiology and Ecology of Health and Disease in Ethiopia, Y. Berhane, H. D. Mariam, and H. Kloos, Eds., Shama Book, Ethiopia, 2006.

[10] B. Roma and S. Worku, "Magnitude of Schistosoma mansoni and intestinal helminthic infections among school children in Wondo Genet Zuria, Southern Ethiopia," Ethiopian Journal of Health Development, vol. 11, pp. 125-129, 1997.

[11] W. T. Michael, T. Endeshaw, T. Shibre et al., "Intestinal parasitic infection in Western Abaya with special reference with Schistosomiasis mansoni," Ethiopian Journal of Health Devevelopment, vol. 13, pp. 21-26, 1999.

[12] Y. Merid, M. Hegazy, G. Mekete, and T. S. Mariam, "Intestinal helmintic infection among children at Lake Awassa Area, south Ethiopia," Ethiopian Journal of Health Development, vol. 15, no. 1, pp. 31-37, 2001.

[13] H. Birrie, C. T. Lo, B. Erko, A. Redda, and N. Gemeda, "Further investigations on freshwater snails of Ethiopia," SINET: Ethiopian Journal of Science, vol. 18, pp. 195-206, 1995.

[14] H. Kloos, C. T. Lo, H. Birrie, T. Ayele, S. Tedla, and F. Tsegay, "Schistosomiasis in Ethiopia," Social Science and Medicine, vol. 26, no. 8, pp. 803-827, 1988.

[15] A. Ali, C. T. Lo, and T. Ayele, "Schistosoma haematobium in Western Ethiopia," Ethiopian medical journal, vol. 24, no. 2, pp. 73-78, 1986.

[16] T. Harrison, "Schistosomiasis and other trematode infection," in Harrison's principles of Internal Medicine, A. Adel and F. Mohamed, Eds., pp. 1266-1271, McGraw-hill, New York, NY, USA, 16th edition, 2005.

[17] N. B. Kabatereine, S. Brooker, E. M. Tukahebwa, F. Kazibwe, and A. W. Onapa, "Epidemiology and geography of Schistosomo mansoni in Uganda: implications for planning control," Tropical Medicine and International Health, vol. 9, no. 3, pp. 372-380, 2004.

[18] A. Assefa, T. Dejenie, and Z. Tamass, "Infection prevalence of schistosoma mansoni and associated risk factors among schoolchildren in suburbs of Mekelle city, Tigray, North Ethiopia," Momona Ethiopian Journal of Science, vol. 5, no. 1, pp. 174-188, 2013.

[19] WHO, Basic Laboratory Methods in Medical Parasitology, World Health Organization, Geneva, Switzerland, 1991.

[20] WHO, "Prevention and control of schistosomiasis and soiltransmitted helminthiasis. Report of WHO expert committee," Tech. Rep., World Health Organization, 2002.

[21] W. Awoke, M. Bedimo, and M. Tarekegn, "Prevalence of schistosomiasis and associated risk factors among students attending at elementary schools in Amibera District, Ethiopia," Open Journal of Preventive Medicinevol, vol. 3, no. 2, pp. 199204, 2013.

[22] T. Grum, "The prevalence of intestinal helminthic infections and associated risk factors among school children in Babile town, Estern Ethiopia," Ethiopian Journal of Health Development, vol. 19, pp. 140-147, 2005.
[23] H. Mitiku, M. Legesse, Z. Teklemariam, and B. Erko, “Transmission of Schistosoma mansoni in Tikur Wuha area, Southern Ethiopia," Ethiopian Journal of Health Development, vol. 24, no. 3, pp. 180-184, 2010.

[24] A. Ayalew, T. Debebe, and A. Worku, "Prevalence and risk factors of intestinal parasites among Delgi school children, North Gondar, Ethiopia," Journal of Parasite and Vector Biologyno, vol. 3 , no. 5 , pp. $75-81$.

[25] L. Jemaneh, "The epidemiology of schistosomiasis mansoni and soil-transmitted helminths in elementary school children from the South Gondar Zone of the Amhara National Regional State, Ethiopia," Ethiopian Medical Journal, vol. 38, no. 2, pp. 105-118, 2000.

[26] T. Essa, Y. Birhane, M. Endris et al., "Current status of schistosoma mansoniand associated risk factors among students in Gorgora Town, northwest, Ethiopia," International Scholarly Research Network Infectious Disease, vol. 2013, Article ID 636103, 7 pages, 2013.

[27] M. Legesse and B. Erko, "Prevalence of intestinal parasires among schoolchildren in a rural area close to the southeast of Lake Langano," Ethiopian Journal of Health Developmentvol, vol. 18, pp. 116-120, 2004.

[28] A. Assefa, T. Dejenie, and Z. Tamass, "Infection prevalence of Schistosoma mansoniand associated risk factors among schoolchildren in suburbs of Mekelle city, Tigray, North Ethiopia," Memona Ethiopian Journal of Science, vol. 5, no. 1, pp. 174-188, 2013.

[29] J. Dejenie and B. Petros, "Irrigation practices and intestinal helminth infections in southern and central zones of Tigray," Ethiopian Journal of Health Development, vol. 23, no. 1, pp. 48$56,2009$.

[30] F. Moges, Y. Belyhun, M. Tiruneh et al., "Intestinal parasite infections in association with cutaneous fungal infection and nutritional status among schoolchildren in Tseda, northwest Ethiopia," Ethiopian Journal Health and Biomed Science, vol. 3, no. 1, pp. 35-43, 2010.

[31] A. Alemu, A. Atnafu, Z. Addis et al., "Soil transmitted helminths and schistosoma mansoni infections among school children in zarima town, northwest Ethiopia," BMC Infectious Diseases, vol. 11, article 189, 2011.

[32] M. Endris, W. Lemma, Y. Belyhun et al., "Prevalence of intestinal parasites and associated risk factors among students of Atse Fasil General Elementary School Azezo, northwest Ethiopia," Ethiopia Journal Health and Biomedical Science, vol. 3, pp. 2533, 2010.

[33] L. Legesse, B. Erko, and A. Hailu, "Current status of intestinal Schistosomiasis and soiltransmitted helminthiasis among primary school children in Adwa Town, Northern Ethiopia," Ethiopian Journal of Health Development, vol. 24, no. 3, pp. 191197, 2010.

[34] T. Handzel, D. M. S. Karanja, D. G. Addiss et al., "Geographic distribution of schistosomiasis and soil-transmitted helminths in Western Kenya: implications for anthelminthic mass treatment," The American journal of tropical medicine and hygiene, vol. 69, no. 3, pp. 318-323, 2003.

[35] R. John, M. Ezekiel, C. Philbert, and A. Andrew, "Schistosomiasis transmission at high altitude crater lakes in Western Uganda," BMC Infectious Diseases, vol. 8, article 110, 2008. 
[36] Center for infectious disease control report, "National prevalence survey on soil-transmitted helminths and schistosomiasis in school children," Rwanda, 2008.

[37] B. Banji, M. Babadoko, G. Mohammed et al., "Survey of Schistosomiasis and other intestinal helminthiases among school-aged children in Agaie, Niger state, Nigeria," Journal of Pharmaceutical and Biomedical Science, vol. 15, no. 7, 2012.

[38] C. S. Barbosa, T. C. Favre, T. N. Wanderley, A. C. Callou, and O. S. Pieri, "Assessment of schistosomiasis, through school surveys, in the Forest Zone of Pernambuco, Brazil," Memorias do Instituto Oswaldo Cruz, vol. 101, no. 1, pp. 55-62, 2006. 

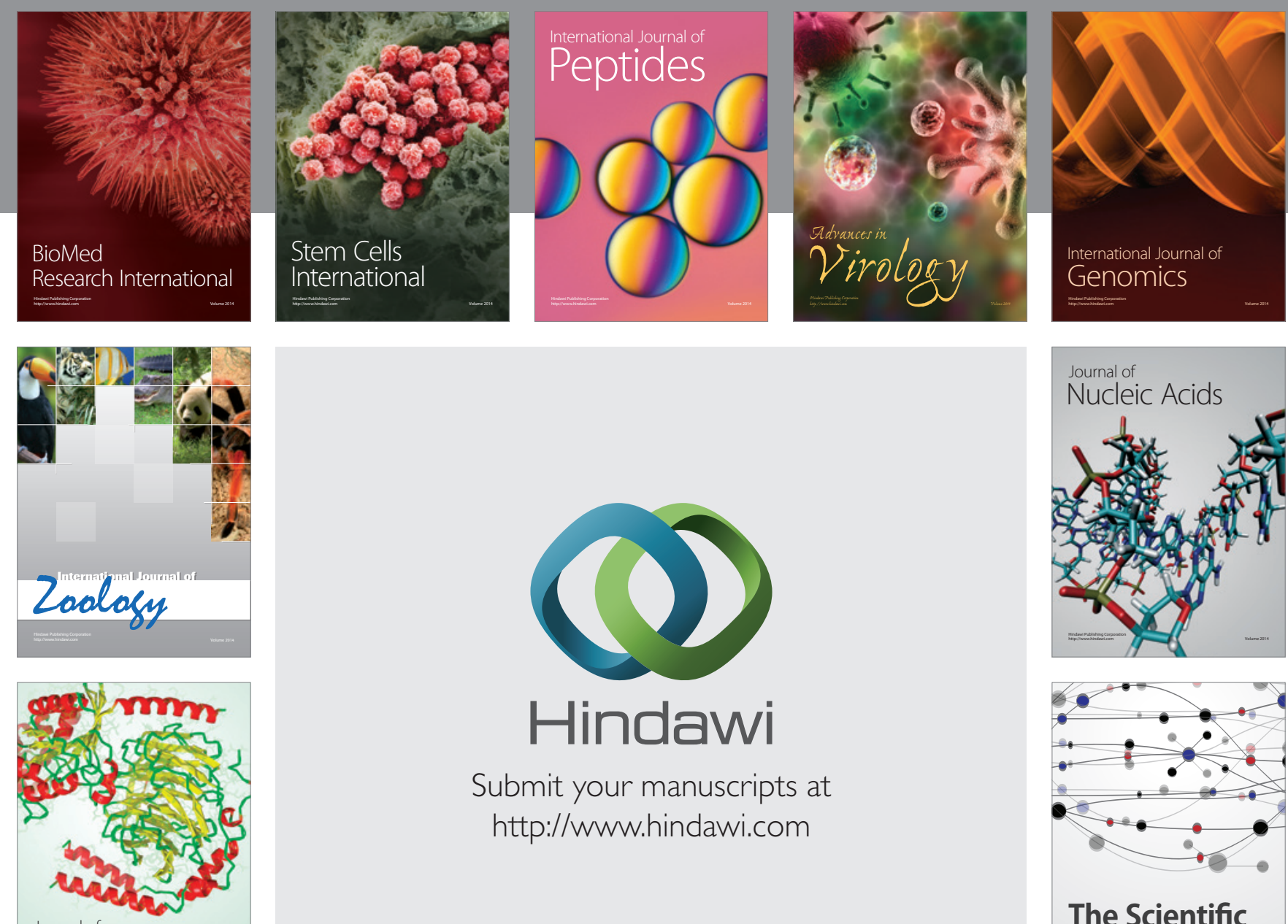

Submit your manuscripts at

http://www.hindawi.com

Journal of
Signal Transduction
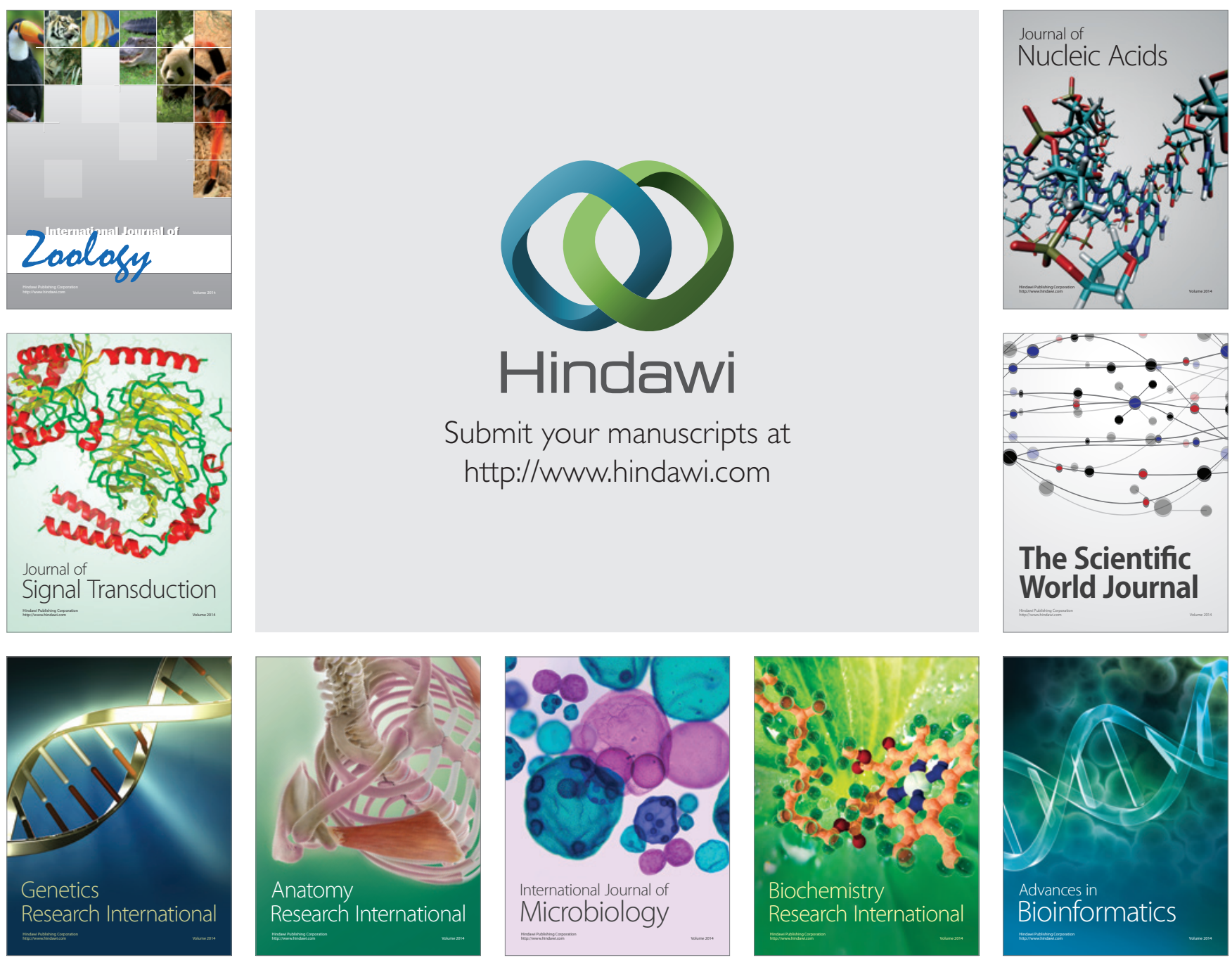

The Scientific World Journal
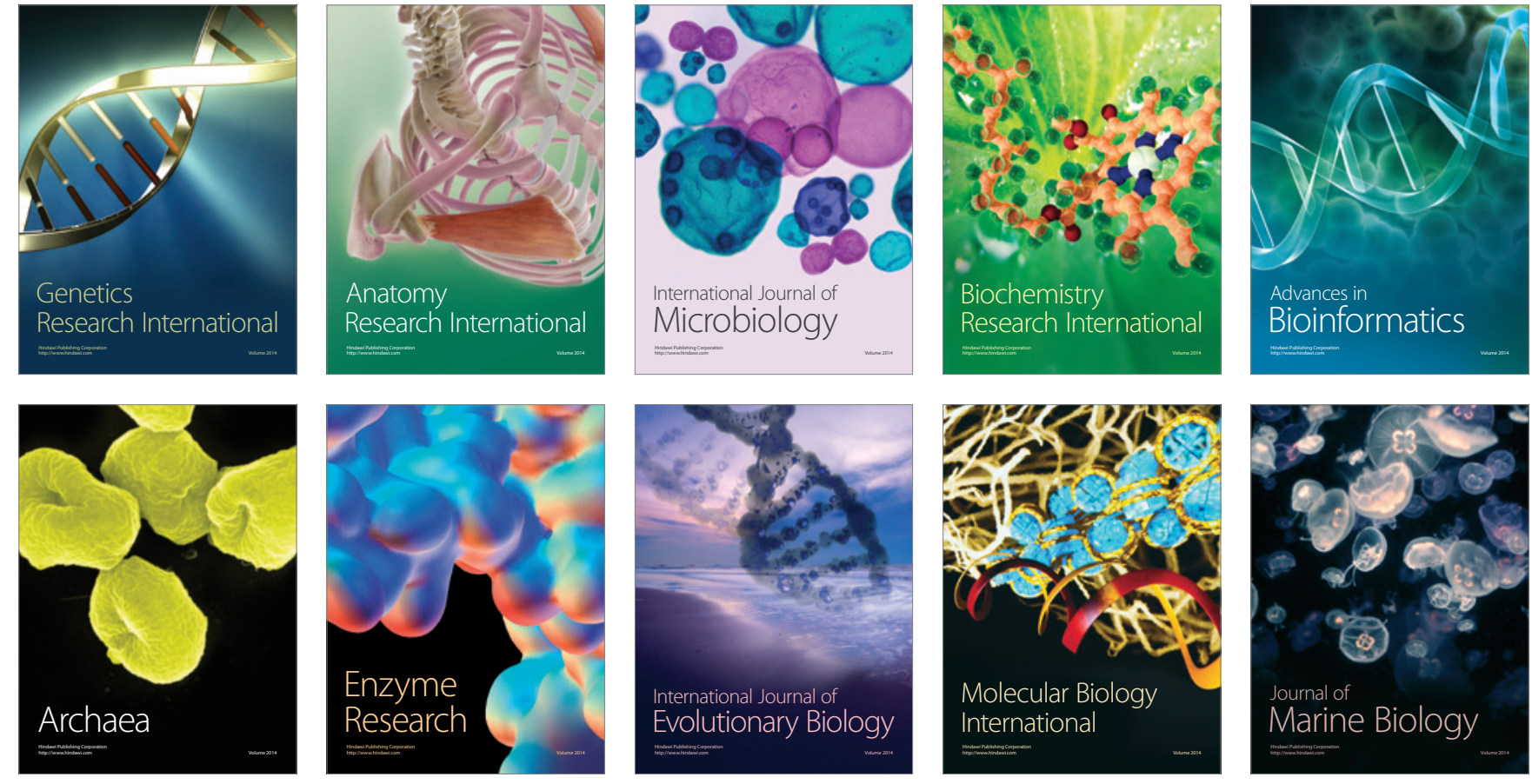